Pacific Journal of Mathematic 


\title{
TRANSFER THEOREMS FOR TOPOLOGICAL STRUCTURES
}

\author{
Fred HaLPERN
}

\begin{abstract}
Transfer theorems are obtained for the following mathematical situations.

$\mathscr{A}$ is a dense substructure of the compact structure $\bar{X}$. $\left\{\mathscr{A}_{2}\right\}$ is the set of all finitely generated substructures of $X$. $F$ is a structure of functions from $Y$ to the structure $X$.

The sentences transferred in the above situations are best described as "almost" positive, variables appearing in a negative subformula are quantified in a prescribed manner.

The main tools of this investigation are the manipulation of classical transfer theorems in the context of commutative diagrams, the ultraproduct conseruction, and the $\mathscr{D}$-limit operation of Chang and Keisler's "Continuous Model Theory."
\end{abstract}

Introduction. Every subgroup of an abelian group is abelian, any extension ring of a ring with zero divisors has zero divisors, and each homomorphic image of a commutative ring is commutative are instances of classical transfer theorems (Lemma 1). In this paper transfer theorems are obtained for the following mathematical situations: $\bar{X}$,

$X$ is a dense substructure of the compact topological structure

$\left\{X_{i}\right\}$ is the set of all finitely generated substructures of $X$, and $F$ is a structure of functions from $Y$ to the structure $X$.

The sentences transferred in the above situations are best described as "almost" positive, variables appearing in a negative subformula of the sentence are quantified in a prescribed manner.

The main tools of this investigation are the manipulation of the classical transfer theorems in the context of commutative diagrams, the ultraproduct construction, and the $\mathscr{D}$-limit operator popularized by Chang and Keisler [3].

Consider a first order language $\mathscr{L}$ and its associated structures. A formula $\sigma$ is identifined with its prenex normal form, i.e., $\sigma$ is assumed to be of the form

$$
Q_{1} v_{1} Q_{2} v_{2}, \cdots, Q_{n} v_{n} M
$$

where each $Q_{i}$ is a quantifier ( $\forall$ or $\exists$ ), each $v_{i}$ is a variable, and $M$ is a formula constructed from (positive) atomic formulae and their negations, negative atomic formulae, using the connectives "and" 
and "or" ( $\wedge$ and $\vee$ ). $Q_{1} v_{1}, \cdots, Q_{n} v_{n}$ is the prefix, and $M$ the matrix, of $\sigma$. A variable or constant term is negative in $\sigma$ if it occurs in some negative atomic formula of $\sigma$. Formulae are classified on the basis of their prefix and matrix.

Assume a formula $\sigma=Q_{1} v_{1} Q_{2} v_{2}, \cdots, Q_{n} v_{n} M . \quad \sigma$ is positive (negative) if its matrix $M$ is constructed solely from positive (negative) atomic formulae. $\sigma$ is universal (existential) if each quantifier of its prefix is a universal (existential) quantifier. $\sigma$ is universalpositive if it is both universal and positive.

Vector notation will be used when convenient. $\sigma\left(v_{1}, \cdots, v_{n}\right)$ is written $\sigma(\vec{v}), \sigma\left(c_{1}, \cdots, c_{n}\right)$ as $\sigma(\vec{c})$, and $\exists v_{1}, \cdots, \exists v_{n} \sigma\left(v_{1}, \cdots, v_{n}\right)$ as $\exists \vec{v} \sigma(\vec{v})$.

Let $\mathscr{A}$ be a structure. $\mathscr{A} \vdash \sigma(\vec{a})$ denotes that the $n$-tuple $\vec{a}$ of elements of $\mathscr{A}$ satisfies the formula $\sigma(\vec{v})$. Let $\varphi$ be a function between the structures $\mathscr{A}$ and $\mathscr{B}$. $\phi$ preserves the $n$-ary operation $F(\vec{v})$ if $F(\varphi(\vec{a}))=\varphi(F(\vec{a}))$ for all $n$-tuples $\vec{a}$ of elements of $\mathscr{A}$. $\varphi$ preserves the formula $\sigma(\vec{v})$ if $\mathscr{A} \vdash \sigma(\vec{a})$ implies $\mathscr{B} \vdash \sigma(\varphi(\vec{a}))$. $\mathscr{B}$ is a homomorphic image of $\mathscr{A}$ if there exists a function $\varphi$ from $\mathscr{A}$ onto $\mathscr{B}$ which preserves all operations and positive atomic formulae. $\sigma(\vec{v})$ is preserved under homomorphic image if $\sigma(\vec{v})$ is preserved by all onto homomorphisms. $\mathscr{A}$ is a substructure of $\mathscr{B}$ (and $\mathscr{B}$ is an extension of $\mathscr{A}$ ) if there exists a $1-1$ function $\varphi$ from $\mathscr{A}$ to $\mathscr{B}$ which preserves the operations, positive atomic formulae, and negative atomic formulae of $\mathscr{A} \cdot \sigma(\vec{v})$ is preserved under substructure if $\mathscr{B} \vdash \sigma(\vec{b})$ and $\varphi(\vec{a})=\vec{b}$ imply $\mathscr{A} \vdash \sigma(\vec{a})$ for every substructure morphism $\varphi: \mathscr{A} \rightarrow \mathscr{B} . \sigma(\bar{a})$ is preserved under extension if $\sigma(\vec{v})$ is preserved by every substructure morphism.

Lemma 1 (Classical Theorems). Let $\sigma$ be a formula.

(i) (Los, Tarski) $\sigma$ is preserved under substructures iff $\sigma$ is universal.

(ii) (Robinson) $\sigma$ is preserved under extensions iff $\sigma$ is existential.

(iii) (Lyndon) $\sigma$ is preserved under homomorphic images iff $\sigma$ is positive.

1. Main results. $\mathscr{A}$ is a topological structure if its underlying set $A$ is endowed with a Hausdorff topology wherein each operation of $\mathscr{A}$ is continuous and wherein each $n$-ary relation of $\mathscr{A}$ is closed as a subset of $A^{n}$; $\mathscr{A}$ is a compact topological structure if its underlying topology if compact. $\mathscr{A}$ is a dense substructure of the topological structure $\overline{\mathscr{A}}$ if $\mathscr{A}$ is a substructure of $\mathscr{A}$ whose underlying set is topologically dense in $\mathscr{A}$. The collection of 
compact structures $\overline{\mathscr{C}}$ compactifies the collection of structures $\mathscr{M}$ if each structure of $\mathscr{A}$ is a dense substructure of a member of It.

The formula $\sigma$ is $\exists$-generalized positive if the prefix of $\sigma$ begins with an initial string of existential quantifiers (i.e., $\sigma=$ $\left.\exists \vec{v} Q_{1} v_{1}, \cdots, Q_{n} v_{n} M\right)$ such that every negative variable of $\sigma$ is free or existentially quantified in the initial string $\exists \vec{v}$. The negation of a $\exists$-generalized positive formula is called a $\forall$-generalized negative formula; it begins with an initial string of universal quantifiers such that each of it nonnegative variables is free or universally quantified in the initial string.

THeORem 1. Let Af be a dense substructure of the compact structure $\overline{\text {. }}$ Every $\exists$-generalized positive sentence satisfied by $\mathscr{A}$ is satisfied by $\overline{\text {. }}$.

This generalizes a result of Robinson [7].

THEOREM 2. Let $\overline{\mathscr{C}}$ compactify the collection of structures $\mathscr{C}$.

(i) Every 3-generalized positive sentence satisfied in $\mathscr{C l}$ is satisfied in $\overline{\mathscr{C}}$.

(ii) Every $\forall$-generalized negative sentence valid in $\mathscr{\mathscr { C }}$ is valid in $\mathscr{L l}$.

CoRollaries to Theorem 2. Let $\sigma$ be a $\forall$-generalized negative sentence (in an appropriate language).

2.1. $\sigma$ is valid for all extensions of the group $\mathscr{G}$ if $\sigma$ is valid for all compact groups which are extensions of $\mathscr{G}$.

2.2. $\sigma$ is valid for all total orderings if $\sigma$ is valid for all complete total orderings.

2.3. $\sigma$ is lvalid for all totally ordered lattices extending the ordering $\mathscr{F}$ if $\sigma$ is valid for all complete totally ordered lattices extending $\mathscr{F}$.

2.4. $\sigma$ is valid for all distributive lattices if $\sigma$ is valid for all complete distributive lattices.

2.5. $\sigma$ is valid for all boolean algebras if $\sigma$ is valid for all complete boolean algebras.

The corollaries to Theorem 2 follow from:

Every group is a dense subgroup of a compact group (Kelly [6], p. 247).

Every total order can be (densely) embedded in a complete total 
order, and every complete total order forms a compact topological space wherein the lattice operations, $\wedge$ and $\vee$, are continuous (Birkhoff [2] pgs. 240-253).

The lattice (boolean algebra) of all subsets of a fixed set $X$ endowed with the interval topology forms a compact topological space wherein the lattice (boolean algebra) operations are continuous. Every distributive lattice is isomorphic to a ring of sets, and every boolean algebra is isomorphic to a field of sets.

Corollary 2.3 considered the total order as a lattice so as to enlarge the class of sentences preserved. The analog of Corollary 2.3 for arbitrary lattices in false. It is difficult to go from complete lattice to compact-Hausdorff space. In fact,

Counterexample 1.1. (Birkhoff [2], pgs. 126-128.) The completion by cuts of the modular lattice of all subspaces of Hilbert space which have finite dimension or codimension is nonmodular (i.e., does not satisfy the modular equation).

Counterexample 1.2. If in Theorem 1 the hypothesis, the topology of $\mathscr{A}$ is compact, is replaced by the topology of $\mathscr{A}$ is a complete metric space, even positive sentences are not preserved.

Let $\sigma=\forall y \exists x\left(y^{2}-2\right) x=1$. Rationals $\vdash \sigma$, but not Reals $\vdash \sigma$.

Counterexample 1.3. It does not seem that the class of sentences preserved under the hypothesis of Theorem 1 can be enlarged. A reasonable conjecture would be the class of all sentences whose negative variables are existentially quantified.

Consider the sentence in the language of order which states no first element exists,

$$
\sigma=\forall \times \exists y \exists z(y \leqq x \text { and } z \leqq y \text { and } z \neq y) .
$$

The open interval $(0,1)$ is dense in $[0,1] ;(0,1) \vdash \sigma$, but not $[0,1] \vdash \sigma$.

The collection of substructures $\left\{\mathscr{A}_{i}\right\}$ generates the structure $\mathscr{A}$ if each finite subset of $\mathscr{A}$ is a subset of some $\mathscr{A}_{i}$. The formula $\sigma$ is $\nabla^{+} \exists$-generalized positive if $\sigma=Q_{1} v_{1}, \cdots, Q_{n} v_{n} \exists \vec{v} M$ (variables of $\exists \vec{v}$ are called terminal) such that each existentially quantified variable is terminal, or is nonnegative and does not appear in any atomic formula in which a terminal variable appears.

A formula $\sigma$ is universal-existential if $\sigma=\forall \vec{w} \exists \vec{v} M$.

THEOREM 4. Let $\left[\mathscr{A}_{i}\right]$ generate the structure $\mathscr{A}$.

(i) (Los and Susco) Any universal-existential sentence valid in $\left\{\mathscr{A}_{i}\right\}$ is satisfied by $\mathscr{A}$. 
(ii) If, in addition, $\mathscr{A}$ is a compact structure, then every $\forall^{+} \exists$-generalized positive sentence valid in $\left\{\mathscr{A}_{i}\right\}$ is satisfied by $\mathscr{A}$.

Corollaries to Theorem 4. Let $\sigma$ be $a \forall^{+} \exists$-generalized positive sentence.

4.1. If $\sigma$ is valid for all finitely generated groups, then $\sigma$ is valid for all compact groups.

4.2. If $\sigma$ is valid for all finite total orderings (viewed as a lattice), then $\sigma$ is valid for all complete totally ordered lattices.

As an application of Corollary 4.2, every totally ordered complete lattice must have a first element. The situation is radically different if we inquire about second elements. We counterexample attempts to weaken conditions on sentences preserved under the hypotheses of Theorem 4 (ii). Examples are taken from the language of total orderings with first element 0 and last element 1.

Counterexample 4.1. Consider the sentence which has a nonterminal existentially quantified negative variable,

$$
\sigma=\exists x \forall y(x \neq 0 \text {, and } x \leqq y \text { or } y=0) .
$$

$\sigma$ asserts the existence of a second element. $\sigma$ is not satisfied by the complete total ordering $[0,1]$, but every finite subset of $[0,1]$ satisfies $\sigma$.

Counterexample 4.2. Consider the sentence which has a nonterminal (nonnegative) existentially quantified variable which appears in an atomic formula with a terminal variable,

$$
\sigma=\exists x \forall y \exists z(z=x \text { and } z \neq 0 \text {, and } y=0 \text { or } z \leqq y) .
$$

Again, $\sigma$ asserts the existence of a second element. [0,1] doesn't satisfy $\sigma$, but each finite subset does satisfy $\sigma$.

Let $\mathscr{A}$ be a structure, $\mathscr{A}^{I}$ denotes the product structure whose underlying set is the set of all functions from $I$ to $\mathscr{A}$ such that operations on functions of $\mathscr{A}^{I}$ are performed coordinatewise and, such that an atomic relation holds in $\mathscr{A}^{I}$ iff it holds in each coordinate. $F$ is a functional structure of range $\mathscr{A}$ if $F$ is a substructure of $\mathscr{A}^{I}$ which contains all constant functions. The functional structure $F \subset \mathscr{A}^{I}$ has finite solution property if, for all unequal $f, f^{\prime} \in F$, $\left\{i \in I \mid f(i)=f^{\prime}(i)\right\}$ is finite.

The formula $\sigma$ is $\forall^{+}$-generalized positive if no negative variable of $\sigma$ is existentially quantified. 
THEOREM 5. Let $F$ be a functional structure of range $\mathscr{A}$.

(i) Every $\forall^{+}$-generalized positive sentence satisfied by $F$ is satisfied by $\mathscr{A}$.

(ii) If, in addition, $F$ has the finite solution property then every $\forall^{+} \exists$-generalized positive sentence satisfied by $F$ is satisfied by $\mathscr{A}$.

The negation of a $\forall^{+}$-generalized positive sentence is a $\exists^{+}$generalized negative sentence; it has the defining property that each variable appearing in a positive atomic formula is existentially quantified.

COROLlARY 5.1. Every $\Xi^{+}$-generalized negative sentence is preserved under direct powers.

COROLlary 5.2. (i) The ring of real polynomials in one variable is functional over the ring of real numbers, with finite solution property.

(ii) Theorem 5 (ii) may be applied to the ring of functions analytic in the circle, $|\boldsymbol{z}|<1$.

(iii) Let $\mathscr{R}$ be a ring and $M$ and $M^{\prime} \mathscr{R}$-modules. The module of homomorphisms from $M$ to $M^{\prime}$ is a functional structure of range $M^{\prime}$.

If, in addition, $M$ is irreducible (has no nontrivial submodules), the above module of homomorphisms has the finite solution property.

2. Proof of theorems. The function $\varphi$ from $\mathscr{A}$ to $\mathscr{B}$ is an elementary embedding if, $\mathscr{A} \vdash \sigma(\vec{a})$ implies $\mathscr{B} \vdash \sigma(\varphi(\vec{a}))$ for all formulae $\sigma(\vec{v})$.

Throughout this section $I$ denotes a fixed index set. $\mathscr{D}$ is a filter on $I$ if $\mathscr{D}$ is a subset of the power set of $I$, such that

$$
\begin{aligned}
& \text { if } X \in \mathscr{D} \text { and } X \subseteq Y \subseteq I \text {, then } Y \in \mathscr{D} \text {, and } \\
& \text { if } X, Y \in \mathscr{D} \text { then } X \cap Y \in \mathscr{D} \text {. }
\end{aligned}
$$

An ultrafilter on $I$ is a maximal filter on $I$, i.e., a filter $\mathscr{D}$ on $I$ such that, for all $X \subseteq I, X \in \mathscr{D}$ or $I-X \in \mathscr{D} . \prod_{i \in I} X_{i}\left(\prod_{i \in I} \mathscr{X}_{i}\right)$ denotes the cartesian product of the sets $\left\{X_{i}\right\}_{i \in I}$ (of the structures $\left.\left\{\mathscr{A}_{i}\right\}_{i \in I}\right)$. When $\forall i \in I X_{i}=X, \prod_{i \in I} X_{i}$ is written $X^{I}$, and similarly for structures. $X^{I}$ can be identified with the set of all functions from $I$ to $X$; an embedding diag: $X \rightarrow X^{I}$ is obtained by identifying $x \in X$ with the function with constant value $x$. For $f \in \prod_{i \in I} X_{i}, f(i)$ denotes the $i$ th coordinate of $f$. 
Assume a set of structures $\left\{\mathscr{A}_{i}\right\}_{i \in I}$ and an ultrafilter $\mathscr{D}$ on $I$. The ultraproduct of $\left\{\mathscr{X}_{i}\right\}_{i \in I}$ by $\mathscr{D}$, written $\Pi \mathscr{A}_{i} \mid \mathscr{D}$, is the homomorphic image of the product structure $\prod_{i \in I} \mathscr{A}_{i}$ induced by the congruence relation

$$
f \equiv f^{\prime} \text { iff }\left\{i \mid f(i)=f^{\prime}(i)\right\} \in \mathscr{D},\left(f \text { and } f^{\prime} \in \Pi \mathscr{A}_{i}\right) .
$$

In the case $\forall i \in I \mathscr{A}_{i}=\mathscr{A}$, the ultraproduct is called the ultrapower of $\mathscr{A}$ by $\mathscr{D}$ and is written $\mathscr{A}^{I} / \mathscr{D}$. Members of $\Pi \mathscr{A}_{i} / \mathscr{D}$ are written $f / \mathscr{D}, f \in \Pi \mathscr{L}_{i}$.

Lemma 2 (Los). The diagonal embedding diag: $\mathscr{A} \rightarrow \mathscr{A}^{\mathrm{I}} / \mathscr{D}$ is an elementary embedding.

Definitions. (i) $\mathscr{A}$ is the outside of a $\mathscr{B}$-sandwich if there exist functions

$$
\mathscr{A} \stackrel{\varphi}{\longrightarrow} \mathscr{B} \stackrel{\psi}{\longrightarrow} \mathscr{A}^{I} / \mathscr{D}
$$

such that $\varphi$ is a substructure morphism, $\psi$ is a substructure morphism, and

$$
\varphi \circ \psi=\operatorname{diag}: \mathscr{A} \longrightarrow \mathscr{A}^{I} / \mathscr{D} \text {. }
$$

(ii) $\mathscr{A}$ is a retract of $\mathscr{B}$ if there exist functions

$$
\mathscr{4} \stackrel{\varphi}{\longleftarrow} \mathscr{B}
$$

such that $\varphi$ is a substructure morphism, $\psi$ is an onto homomorphism, and $\varphi \circ \psi=$ identity of $\mathscr{A}$.

(iii) $\mathscr{A}$ is a strong retract of $\mathscr{B}$ if there exists functions

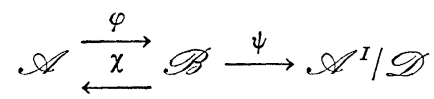

such that $\mathscr{A}$ is the outside of a $\mathscr{B}$-sandwich by $\mathscr{A} \stackrel{\varphi}{\rightarrow} \mathscr{B} \stackrel{\psi}{\rightarrow} \mathscr{A}^{I} / \mathscr{D}$ and $\mathscr{A}$ is a retract of $\mathscr{B}$ by

$$
\mathscr{L} \stackrel{\varphi}{\stackrel{\chi}{\longleftarrow}} \mathscr{B}
$$

(iv) $\mathscr{B}$ is an ultrahomomorphic image of $\mathscr{A}$ is there exists a commutative diagram 


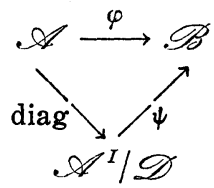

such that $\varphi$ is a substructure morphism and $\psi$ an onto homomorphism.

THEOREM 6. Let $\sigma(\vec{v})$ be a formula, $\mathscr{A}$ a substructure of $\mathscr{B}$ by $\mathscr{\varphi} \stackrel{\varphi}{\longrightarrow} \mathscr{B}$, and $\bar{a}$ an n-tuple of elements of $\mathscr{A} . \vec{a}$ shall also denote the n-tuple of elements of $\mathscr{B}, \quad \overrightarrow{\varphi(a)} . \mathscr{B} \vdash \sigma(\bar{a})$ implies $\mathscr{A} \vdash \sigma(\bar{a})$ if

(i) (Keisler) $\mathscr{A}$ is the outside of a $\mathscr{B}$-sandwich and $\sigma$ is a universal-existential formula.

(ii) (Keisler) $\mathscr{A}$ is a retract of $\mathscr{B}$ and $\sigma$ is a $\forall^{+}$-generalized positive formula.

(iii) $\mathscr{A}$ is a strong retract of $\mathscr{B}$ and $\sigma$ is a $\forall^{+} \exists$-generalized positive sentence.

(iv) If $\mathscr{B}$ is an ultrahomomorphic image of $\mathscr{A}$ and $\sigma$ is a ヨ-generalized positive sentence, then $\mathscr{A} \vdash \sigma(\vec{a})$ implies $\mathscr{B} \vdash \sigma(\vec{a})$.

Proof. Results follow by induction on the number of quantifiers in $\sigma(\vec{v})$. We assume our language has constants for each element of $\mathscr{A},\{a\}_{a \in \mathscr{A}}$, and constants for each element of $\mathscr{B},\{b\}_{b \in \mathscr{S}}$. The interpretation of constants will be specified in each situation.

(i) Let $\mathscr{A} \stackrel{\varphi}{\longrightarrow} \mathscr{B} \stackrel{\psi}{\longrightarrow} \mathscr{H}^{I} / \mathscr{D}$ be a $\mathscr{B}$-sandwich.

The constant symbol $a$ is interpreted as $\varphi(a)$ in $\mathscr{B}$ and as $\alpha \circ \beta(a)=\operatorname{diag}(a)$ in $\mathscr{A}^{I} / \mathscr{D}$ (it is here that we use $\alpha \circ \beta=\operatorname{diag}$, to obtain an unambiguous interpretation of $a$ in $\mathscr{A}_{I}(\mathscr{D}) . \quad b$ is interpreted as $\psi(b)$ in $\mathscr{A}^{I} / \sigma$ and is not assigned an interpretation in $\mathscr{A}$.

Assume $\mathscr{B}$ satisfies a universal-existential formula, $\mathscr{B} \vdash \forall \vec{w} \exists \vec{v} M(\vec{w}, \vec{v}, \vec{a})$. We show that $\mathscr{A}$ satisfies same formula. It suffices to show that, for each $n$-tuple $\vec{a}^{\prime}$ of elements of $\mathscr{A}$,

$$
\mathscr{A} \vdash \exists \vec{v} M\left(\vec{a}^{\prime}, \vec{v}, \vec{a}\right) \text {. }
$$

Clearly, $\mathscr{B} \vdash \exists \vec{v} M\left(\vec{a}^{\prime}, \vec{v}, \vec{a}\right)$. Thus, for some $\vec{b}, \mathscr{B} \vdash M\left(\vec{a}^{\prime}, \vec{b}, \vec{a}\right)$.

Since $\mathscr{B}$ is a substructure of $\mathscr{A}^{I} / \mathscr{D}, \mathscr{A}_{1} / \mathscr{D} \vdash M\left(\vec{a}^{\prime}, \vec{b}, \vec{a}\right)$. Thus $\mathscr{A}^{I} / \mathscr{D} \vdash \exists \vec{v} M\left(\vec{a}^{\prime}, \vec{v}, \vec{a}\right)$. Hence, $\mathscr{A} \vdash \exists \vec{v} M\left(\vec{a}^{\prime}, \vec{v}, \vec{a}\right)$.

(ii) Let

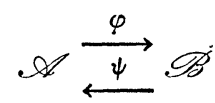

be a retraction.

$\alpha$ is interpreted as $\varphi(\alpha)$ in $\mathscr{B}$ (note that, since $\varphi \circ \psi=$ identity 
of $\mathscr{A}$, the interpretation of $a$ in $\mathscr{A}$ is unambiguous). $b$ is interpreted as $\psi(b)$ in $\mathscr{A}$.

Consider a $\forall^{+}$-generalized positive sentence $\sigma(\vec{a}, \vec{b})$ in which no constant of $\vec{b}$ is negative. We show that

$$
\mathscr{B} \vdash \sigma(\vec{a}, \vec{b}) \text { implies } \mathscr{A} \vdash \sigma(\vec{a}, \vec{b}) \text {. }
$$

(1) is trivial if $\sigma(\vec{a}, \vec{b})$ has no quantifiers.

Now consider $\sigma(\vec{a}, \vec{b}, v)$ where no constant of $\vec{b}$ is negative.

Assume that $\forall b \in \mathscr{B}$, (1) holds for $\sigma(\vec{a}, \vec{b}, b)$.

If $\mathscr{P} \vdash \forall v \sigma(\vec{a}, \vec{b}, v)$, then $\forall a \in \mathscr{A}, \mathscr{B} \vdash \sigma(\vec{a}, \vec{b}, a)$. By induction hypothesis, $\forall a \in \mathscr{A}, \mathscr{A} \vdash \sigma(\bar{a}, \vec{b}, a)$. Thus $\mathscr{A} \vdash \forall v \sigma(\vec{a}, \vec{b}, v)$. This completes the first part of our induction.

Now assume that $v$ is not a negative variable of $\sigma(\vec{a}, \vec{b}, v)$ and assume $\mathscr{B} \vdash \exists v \sigma(\vec{a}, \vec{b}, v)$. For some $b \in \mathscr{B}, \mathscr{B} \vdash \sigma(\vec{a}, \vec{b}, b)$. Since $v$ was not a negative variable, $b$ does not appear negatively in $\sigma(\vec{a}, \vec{b}, b)$ and hence $\sigma(\vec{a}, \vec{b}, b)$ falls under the induction hypothesis. Thus $\mathscr{A} \vdash \sigma(\vec{a}, b, b)$ which implies $\mathscr{A} \vdash \exists v \sigma(\bar{a}, \vec{b}, v)$. This completes the induction.

We shall prove (iv) and then prove the difficult (iii).

We assume a language with special symbols for true and false, $t$ and $f$; we will also assume these symbols may be subscripted by a formula $\sigma, t_{\sigma}$ and $f_{\sigma} . \quad p_{\sigma}$ shall vary over $\left\{t_{\sigma}, f_{\sigma}\right\} . \sigma[\varphi / \psi]$ denotes the formula resulting from replacing each occurrence of the formula $\phi$ in $\sigma$ by $\psi$; and similarly for finite sets of formulae $\Phi, \sigma[\varphi / \psi]_{\varphi \in \Phi}$.

(iv) Assume the ultrahomomorphism

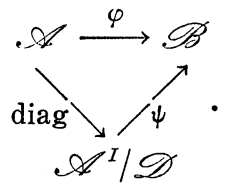

$a$ is interpreted as $\varphi(a)=(\operatorname{diag} \circ \psi)$ (a) in $\mathscr{B} . \quad b$ is not interpreted.

Let $\exists \vec{v} \sigma(\vec{a}, \vec{v})$ be a $\exists$-generalized sentence, i.e., $\sigma(\vec{a}, \vec{v})$ has no negative constants or variables other than those of $\vec{a}$ and $\vec{v}$. Note that $\sigma(\vec{a}, \vec{v})$ may contain other quantified nonnegative variables. Assume $\mathscr{A} \vdash \exists \vec{v} \sigma(\vec{a}, \vec{v})$. We show $\mathscr{B} \vdash \exists \vec{v} \sigma(\vec{a}, \vec{v})$. $\mathscr{A} \vdash \exists \bar{v} \sigma(\vec{a}, \vec{v})$ implies $\mathscr{A} \vdash \sigma\left(\vec{a}, \vec{a}^{\prime}\right)$ for some $\vec{a}^{\prime}$. Let $N_{\sigma}$ denote the set of negative atomic formulae appearing in $\sigma\left(\vec{a}, \vec{a}^{\prime}\right)$. By the form of $\sigma(\vec{a}, \vec{v})$, each formula $\alpha$ of $N_{\sigma}$ has no variables and contains only the constants of $\vec{a}$ and $\vec{a}^{\prime}$.

$$
\forall \alpha \in N_{o}, \mathscr{A} \vdash \alpha \text { iff } \mathscr{B} \vdash \alpha
$$


Write $p_{\alpha}=t_{\alpha}$ if $\mathscr{A} \vdash \alpha ; p_{\alpha}=f_{\alpha}$ otherwise $\left(\alpha \in N_{\sigma}\right)$, and define $\sigma^{*}\left(\vec{a}, \bar{a}^{\prime}\right)=\sigma\left(\vec{a}, \vec{a}^{\prime}\right)\left[\alpha / p_{\alpha}\right]_{\alpha \in N_{\sigma}}$. Since $\mathscr{A}$ is a substructure of $\mathscr{B}$ and $\mathscr{A} \vdash \alpha \leftrightarrow p_{\alpha}$,

$$
\mathscr{B} \vdash \alpha \longleftrightarrow p_{\alpha}
$$

Now, $\mathscr{A} \vdash \sigma^{*}\left(\vec{a}, \vec{a}^{\prime}\right)$ whence $\mathscr{A}^{I} / \mathscr{D} \vdash \sigma^{*}\left(\vec{a}, \vec{p}^{\prime}\right)$. Also $\sigma^{*}\left(\vec{a}, \vec{a}^{\prime}\right)$ is a positive sentence. $\mathscr{B} \vdash \sigma^{*}\left(\vec{a}, \bar{a}^{\prime}\right)$ by Lemma 1 (iii) since $\mathscr{B}$ is a homomorphic image of $\mathscr{A}^{I} / \mathscr{D}$.

$\mathscr{B} \vdash \sigma^{*}\left(\vec{a}, \vec{a}^{\prime}\right) \quad\left[p_{\alpha} / \alpha\right]_{\alpha \in N_{\sigma}}$ by $\quad$ (3). Thus, $\mathscr{B} \vdash \sigma\left(\vec{a}, \vec{a}^{\prime}\right)$ and $\mathscr{B} \vdash \exists \bar{v} \sigma(\vec{a}, \vec{v})$.

(iii) Let $\mathscr{A} \stackrel{\varphi}{\rightarrow} \mathscr{B} \stackrel{\psi}{\rightarrow} \mathscr{A}^{I} / \mathscr{D}, \mathscr{B} \stackrel{\chi}{\rightarrow} \mathscr{A}$ be a strong retract.

$a$ is interpreted as $\varphi(a)$ in $\mathscr{B}$ and as $\varphi \circ \psi=\operatorname{diag}(a)$ in $\mathscr{A}^{I} / \mathscr{D}$.

$b$ is interpreted as $\psi(b)$ in $\mathscr{A}^{I} / \mathscr{D}$ and as $\chi(b)$ in $\mathscr{A}$. To emphasize interpretation in $\mathscr{A}$ (and to avoid confusion), $\chi(b)$ is written $\hat{b}$.

Let $\sigma(\vec{a}, \vec{b})=Q_{1} v_{1}, \cdots, Q_{n} v_{n} \exists \vec{v} M(\vec{a}, \vec{b}, \vec{v})$ be such that

No existentially quantified variable of

$\left\langle v_{1}, \cdots, v_{n}\right\rangle$ and no constant of $\vec{b}$ is negative or appears in any atomic formula with a variable of $\vec{v}$.

$$
\mathscr{B} \vdash \sigma(\bar{a}, \vec{b}) \text { implies } \mathscr{A} \vdash \sigma(\vec{a}, \overrightarrow{\hat{b}}) \text {, notice } \overrightarrow{\hat{b}} \text {, }
$$

is shown by induction on the length of $Q_{1} v_{1}, \cdots, Q_{n} v_{n}$.

If $n=0, \quad \sigma(\vec{a}, \vec{b})$ is of the form $\exists \vec{v} M(\vec{a}, \vec{b}, \vec{v})$. Assume $\mathscr{B} \vdash \exists \vec{v} M(\vec{a}, \vec{b}, \vec{v})$. Set $A_{M}=$ the set of positive atomic formulae of $\exists \vec{v} M(\vec{a}, \vec{b}, \vec{v})$ in which variables $\vec{v}$ do not appear. $\forall \alpha \in A_{M}$ write $p_{\alpha}=t_{\alpha}$ if $\mathscr{B} \vdash \alpha(\vec{a}, \vec{b}) ; p_{\alpha}=f_{\alpha}$ otherwise, and define

$$
\exists \vec{v} M^{*}(\vec{a}, \vec{v})=\exists \vec{v} M(\vec{a}, \vec{b}, \vec{v})\left[\alpha / p_{\alpha}\right]_{\alpha \in A_{M}} .
$$

Now, $\mathscr{B} \vdash \exists \vec{v} M^{*}(\vec{a}, \vec{v})$ and no constant of $\vec{b}$ occurs in $\exists \vec{v} M^{*}(\vec{a}, \vec{v})$ by (4). $\mathscr{H}^{I} / \mathscr{D} \vdash \exists \vec{v} M^{*}(\vec{a}, \vec{v})$ since $\mathscr{A}^{I} / \mathscr{D}$ is an extension of $\mathscr{B}$. Thus, $\mathscr{A} \vdash \exists \vec{v} M^{*}(\vec{a}, \vec{v})$ as no variable of $\vec{b}$ occurs in $M^{*}(\vec{a}, \vec{v})$. Consider $\exists \vec{v} M^{*}(\vec{a}, \vec{v})\left[p_{\alpha} / \alpha\right]_{\alpha \in A_{M}}$. If $\mathscr{B} \vdash \alpha(\vec{a}, \vec{b})$, then $\mathscr{A} \vdash \alpha(\vec{a}, \vec{b})$ since $\alpha(\vec{a}, \vec{b})$ is positive and $\mathscr{A}$ is a homomorphic image of $\mathscr{B}\left(\alpha(\vec{a}, \vec{b}) \in A_{M}\right)$. Thus, if $\mathscr{B} \vdash \alpha(\vec{a}, \vec{b})$ (i.e., $\left.p_{\alpha}=t_{\alpha}\right)$, then $\mathscr{A} \vdash \exists \vec{v} M^{*}(\vec{a}, \vec{v})\left[p_{\alpha} / \alpha(\vec{a}, \vec{b})\right]$. Now, if not $\mathscr{B} \vdash \alpha(\bar{a}, \vec{b})$, then $p_{\alpha}=f_{\alpha}$ and it does no harm (by the form of $\left.M^{*}\right)$ to replace $f_{\alpha}$ by $\alpha(\vec{a}, \hat{b})$. Thus $\mathscr{A} \vdash \exists v M^{*}(\vec{a}, \vec{v})\left[p_{\alpha} / \alpha\right]_{\alpha \in A_{M}}$. Thus, $\mathscr{A} \vdash \exists v M(\vec{a}, \vec{b}, \vec{v})$.

We complete the induction.

Let $\forall v \sigma(\vec{a}, \vec{b}, v)$ obey (4) and let $\mathscr{B} \vdash \forall v \sigma(\vec{a}, \vec{b}, v) . \quad \forall a \in$ $\mathscr{A}, \mathscr{B} \vdash \sigma(\vec{a}, \vec{b}, a)$ and $\sigma(\vec{a}, \vec{b}, a)$ obeys (4). Thus by induction 
hypothesis, $\forall a \in \mathscr{A}, \mathscr{A} \vdash \sigma(\vec{a}, \vec{b}, a)$. Hence $\mathscr{A} \vdash \forall v \sigma(\vec{a}, \vec{b}, v)$.

Let $\exists v \sigma(\vec{a}, \vec{b}, v)$ obey (4) and let $\mathscr{B} \vdash \exists v \sigma(\vec{a}, \vec{b}, v)$. For some $b \in \mathscr{B}, \mathscr{B} \vdash \sigma(\vec{a}, \vec{b}, b)$ where $\sigma(\vec{a}, \vec{b}, b)$ obeys (4) since $\exists v \sigma(\vec{a}, \vec{b}, v)$ obeys (4). By induction hypothesis, $\mathscr{A} \vdash \sigma(\vec{a}, \vec{b}, \hat{b})$. Thus $\mathscr{A} \vdash \exists v \sigma(\vec{a}, \vec{b}, v)$.

We prove Theorems 1-5 by showing that each situation yields a diagram to which Theorem 6 applies. Let I be a set. $S_{\omega}(I)$ denotes the set of all finite subsets of $I . \mathscr{D}$ is a regular ultrafilter on $S_{\omega}(I)$ if $\mathscr{D}$ is an ultrafilter on $S_{\omega}(I)$ such that

$$
\forall i_{0} \in I,\left\{S \in S_{\omega}(I) \mid i_{0} \in S\right\} \in \mathscr{D} .
$$

It follows that $\left\{S \in S_{\omega}(I) \mid T \subset S\right\} \in \mathscr{D}$ for each finite $T \subset I$.

Assume a topological space $X$ and ultrafilter $\mathscr{D}$ on $I$. For each $x \in X$ and $f \in X^{I}, x=\mathscr{D}$-limit $f$ if $\left\{i \mid f(i) \in V_{x}\right\} \in \mathscr{D}$ for each open set $V_{x}$ containing $x$.

Essentially the $\mathscr{D}$-lim operator on sequences $f \in X^{I}$ acts like the limit operation on countable sequences, with the added property that, if $X$ is a compact space, $\mathscr{D}$ - $\lim f$ always exists. The properties of the $\mathscr{D}$-limit operator are listed below. A more complete teatment is provided in Chang and Keisler [3], pgs. 6-15.

Let $X$ be a topological space with basis $\delta$ (i.e., for each open set $\left.V, V=\bigcup_{\substack{0 \subseteq \delta \\ 0 \subseteq V}} 0\right)$ and let $\mathscr{D}$ be an ultrafilter on $I . f$ will vary over elements of $X^{I}$.

$\mathscr{D}-L 0 \quad \mathscr{D}-\lim f$ is unique.

$\mathscr{D}-L 1$ If $\left\{i \mid f(i)=f^{\prime}(i)\right\} \in \mathscr{D}$, then $\mathscr{D}-\lim f=\mathscr{D}-\lim f^{\prime} \quad$ (or both don't exist).

$\mathscr{D}-L 2 \quad \mathscr{D}-\lim \left\langle f_{1}, \cdots, f_{n}\right\rangle=\left\langle\mathscr{D}-\lim f_{1}, \cdots, \mathscr{D}-\lim f_{n}\right\rangle$.

$\mathscr{D}-L 3 \mathscr{D}-\lim f$ belongs to the closure (in $X$ ) of the range of $f$, (range $f=\{x \in X \mid \exists i \in I, f(i)=x\}$ ).

$\mathscr{D}-L 3^{\prime}$ The $\mathscr{D}$-limit of a constant sequence equals that constant value.

$\mathscr{D}-L 4$ If $F$ is a continuous function from $X$ to $Y$, then $\mathscr{D}$ $\lim F(f)=F(\mathscr{D}-\lim f)$.

$\mathscr{D}-L 4^{\prime}$ If $R$ is a closed $n$-ary relation and $\forall i \in I, R\left(f_{1}(i), \cdots, f_{n}(i)\right)$, then $R\left(\mathscr{D}-\lim f_{1}, \cdots, \mathscr{D}-\lim f_{n}\right)$.

$\mathscr{D}-L 5$ If $X$ is a compact space, then $\forall f \in X^{I} \mathscr{D}$ - $\lim f$ exists.

$\mathscr{D}-L 6$ If $\mathscr{D}$ is a regular ultrafilter on $I=S_{\omega}(\delta), \delta$ a basis for $\bar{X}$, then, for each set $S \subseteq \bar{X}$ and limit point $x_{0}$ of $S$, there exists a sequence $f \in S^{I}$ such that $\mathscr{D}-\lim f=x_{0}$.

$\mathscr{D}-L 6^{\prime}$ If $X$ is dense in $\bar{X}$, for each $x_{0} \in \bar{X}$, there exists an $X$ sequence $f \in X^{I}$ such that $\mathscr{D}-\lim f=x_{0}$ (i.e., the map $X^{I} \stackrel{\mathscr{D}-\lim }{\longrightarrow} \bar{X}$ is onto). 
THEOREM 7. Let $\mathscr{A}$ be a dense substructure, of the compact structure $\overline{\mathscr{A}}, \mathscr{A} \stackrel{\varphi}{\rightarrow} \mathscr{A} . \quad$ For some set $I$ and ultrafilter $\mathscr{D}$ on $I, \mathscr{A}$ is a homomorphic image of $\mathscr{A}^{I} / \mathscr{D}$ (via the map $\eta$ ) such that

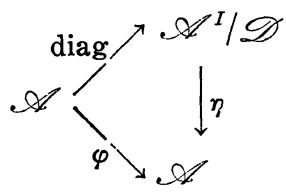

commutes.

Proof. Let $\mathscr{\mathscr { A }}$ have basis $\delta$. Let $\mathscr{D}$ be any regular ultrafilter on $I=S_{\omega}(\delta)$.

Define the function $\eta: \mathscr{A}^{1} / \mathscr{D} \rightarrow \mathscr{A} \quad$ by $\eta(f / \mathscr{D})=\mathscr{D} \lim f$. Since $\mathscr{A}$ is compact, by $\mathscr{D}-L 5, \eta$ is defined $\forall f / \mathscr{D} \in \mathscr{A}^{T} / \mathscr{D} . \eta$ is well defined by $\mathscr{D}-L 1, \eta$ is a homomorphism of structures by $\mathscr{D}-L 4$ and $\mathscr{D}-L 4^{\prime}$, and $\eta$ is onto by $\mathscr{D}-L 6^{\prime}$. Finally, by $\mathscr{D}-L 3^{\prime}$, the diagram of Theorem 7 commutes.

\section{THEOREM 8.}

(i) If $\mathscr{A}$ is a dense substructure of the compact topological structure $\mathscr{A}$, then $\mathscr{A}$ is an ultrahomomorphism of $\mathscr{A}$.

(ii) If $\left\{\mathscr{A}_{j}\right\}$ generate $\mathscr{A}$, then $\mathscr{A}$ is the outside of a $\prod_{i \in I} \mathscr{A}_{2} \mid \mathscr{D}$-sandwich for some ultraproduct $\Pi_{i \in I} \mathscr{A}_{i} \mid \mathscr{D}$.

If, in addition, $\mathscr{A}$ is a compact structure, then $\mathscr{A}$ is a strong retract of $\Pi_{i \in I} \mathscr{X}_{i} \mid \mathscr{D}$.

(iii) If $F \subseteq \mathscr{A}^{I}$ is a functional structure of range $\mathscr{A}$, then $\mathscr{A}$ is a retract of $F$.

If, in addition, $F$ has the finite solution property, then $\mathscr{A}$ is a strong retract of $F$.

Proof. (i) follows from Theorem 7.

(ii) Let $\{\mathscr{A}\}_{j \in J}$ generate $\mathscr{A}$. Let $I=S_{\omega}(\mathscr{A})$ and $\mathscr{D}$ be a regular ultrafilter on $I$. Assign to each $i \in S_{\omega}(\mathscr{A})$ some structure $\mathscr{A}_{i}$ from $\left\{\mathscr{X}_{j}\right\}_{j \in J}$ such that $i \subset \mathscr{A}_{i}$. Consider $\Pi_{i \in I} \mathscr{A}_{i} \mid \mathscr{D}$.

$\mathscr{A}$ is a substructure of $\Pi_{i \in I} \mathscr{A}_{i} / \mathscr{D}$ by the map $\varphi$, where $\varphi(a)=f / \mathscr{D}$ such that $f(i)=a$ if $a \in i$ and $f(i)$ is arbitrary otherwise. Also, since $\mathscr{A}_{i} \subset \mathscr{A}, \prod_{i \in I} \mathscr{A}_{i} / \mathscr{D} \subset \mathscr{A}^{I} / \mathscr{D}$.

Finally, if $\mathscr{A}$ is a compact structure, then $\mathscr{D}$-lim is a map from $\mathscr{A}^{I} / \mathscr{D}$ to $\mathscr{L}$. Since $\prod_{i \in I} \mathscr{\mathscr { A } _ { i }} / \mathscr{D}$ contains all constant functions, the restriction of $\mathscr{D}$-lim to $\Pi_{i \in I} \mathscr{A}_{i} / \mathscr{D}$ is onto.

(iii) Since $F$ contains all constant functions the map $\mathscr{A} \stackrel{\varphi}{\rightarrow} F$ is obvious. Choose any $a \in \mathscr{A}$; the projection map $F \stackrel{\psi}{\rightarrow} \mathscr{A}$, defined 
by $\psi(f)=f(a)$, is onto since $F$ contains all constant functions.

If, in addition, $F \subset \mathscr{A}_{i}$ has the finite solution property, then $\left\{\left\{i \mid f(i) \neq f^{\prime}(i)\right\} \mid f \neq f^{\prime} \in F\right\}$ has the finite intersection property and can be expanded to an ultrafilter $\mathscr{D}$ on $I$. Clearly, the canonical $\operatorname{map} F \rightarrow \mathscr{A}^{I} / \mathscr{D}$ is $1-1\left(F \subset \mathscr{A}^{I}\right)$.

Theorems 1-5 readily follow from Theorems 6 and 8 .

Final Remarks. Theorems 1 and 2 apply to dense substructures of compact topological structures. We consider the noncompact case. A sentence $\sigma$ is ( $\exists$-generalized $\forall$ )-positive if $\sigma=$ $\exists \vec{v} \forall \vec{w} M(\vec{v}, \vec{w})$ where $M$ is quantifier-free and every negative variable of $M$ is existentially quantified in $\exists \vec{v}$.

Theorem 3. Let $\mathscr{A}$ be a dense substructure of the (Hausdorff) topological structure $\mathscr{\text { . }}$ Every ( $\exists$-generalized $\forall$ )-positive sentence satisfied by $\mathscr{A}$ is satisfied by $\hat{\mathscr{A}}$.

The proof of Theorem 3 is similar to the proof of Theorem 1 . Instead of a homomorphism from an ultrapower of $\mathscr{A}$ onto $\mathscr{A}$, one obtains a homomorphism from a subset of an ultrapower of $\mathscr{A}$ onto $\mathscr{A}$ (the domain of the homomorphism is the set of all sequences whose $\mathscr{D}$-limits do exist). Theorem 3 then follows from a result of Keisler [5].

LEMma 3 (Keisler). If $\mathscr{A}$ is a substructure of $\mathscr{B}$ such that $\mathscr{B}$ is a homomorphic image of a substructure of an ultrapower of $\mathscr{A}$ such that $\forall a \in \mathscr{A}$ the image of a under the homomorphism $i s a \in \mathscr{B}$, then every ( $\exists$-generalized $\forall$ )-positive sentence satisfied by $\mathscr{A}$ is satisfied by $\mathscr{B}$.

It is also shown in Keisler [5] that the sentences preserved under inverse limits are precisely the negations of ( $\exists$-generalized $\forall$ )positive sentences.

Theorem 6 (iii) and (iv), as well as (i) and (ii), are characterizations, i.e., the classes of sentences described are the set of all sentences preserved under the indicated algebraic operation. Other preservation theorems and concepts may be found in Bell and Slomson [1] and Chang and Keisler [4].

We have just recently been able to extend Theorems 1-4 applied to groups to a weak second-order language where quantification over subgroups and normal subgroups are permitted. One must restrict the notion of subgroups to topologically closed subgroups. These results will appear elsewhere. 


\section{REFERENCES}

1. J. L. Bell and A. B. Slomson, Models and Ultraproducts, North-Holland, Amsterdam, 1969.

2. G. Birkhoff, Lattice Theory, Amer. Math. Soc. Colloq. Publ. vol. 25, third edition, Amer. Math. Soc., New York, N. Y., 1967.

3. C. C. Chang and H. J. Keisler, Continuous Model Theory, Princeton University Press, 1966.

4. - Model Theory, North-Holland, Amsterdam, 1973.

5. H. J. Keisler, Theory of models with generalized atomic formulas, J. Symbolic Logic 25 (1960), 1-26.

6. J. L. Kelley, General Topology, Van Nostrand, Princeton, 1955.

7. A. Robinson, Compactification of groups and rings and nonstandard analysis,

J. Symbolic Logic, 34 (1969), 577-587.

Received December 20, 1974.

Rider College 


\section{PACIFIC JOURNAL OF MATHEMATICS}

\section{EDITORS}

RICHARD ARENS (Managing Editor)

University of California

Los Angeles, California 90024

\author{
R. A. Beaumont \\ University of Washington \\ Seattle, Washington 98105
}

\section{J. DugundjI}

Department of Mathematics University of Southern California Los Angeles, California 90007

D. Gilbarg and J. Milgram

Stanford University

Stanford, California 94305

\section{ASSOCIATE EDITORS}
E. F. BECKENBACH
B. H. NeumanN
F. WOLF
K. YoshidA

\section{SUPPORTING INSTITUTIONS}

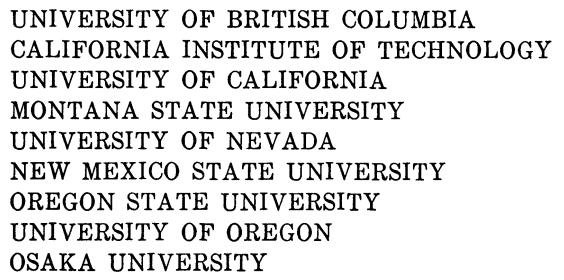

UNIVERSITY OF BRITISH COLUMBIA CALIFORNIA INSTITUTE OF TECHNOLOGY UNIVERSITY OF CALIFORNIA MONTANA STATE UNIVERSITY UNIVERSITY OF NEVADA NEW MEXICO STATE UNIVERSITY OSAKA UNIVERSITY OREGON STATE UNIVERSITY UNIVERSITY OF OREGON

\author{
UNIVERSITY OF SOUTHERN CALIFORNIA \\ STANFORD UNIVERSITY \\ UNIVERSITY OF TOKYO \\ UNIVERSITY OF UTAH \\ WASHINGTON STATE UNIVERSITY \\ UNIVERSITY OF WASHINGTON \\ AMERICAN MATHEMATICAL SOCIETY
}

The Supporting Institutions listed above contribute to the cost of publication of this Journal, but they are not owners or publishers and have no responsibility for its content or policies.

Mathematical papers intended for publication in the Pacific Journal of Mathematics should be in typed form or offset-reproduced, (not dittoed), double spaced with large margins. Please do not use built up fractions in the text of your manuscript. You may however, use them in the displayed equations. Underline Greek letters in red, German in green, and script in blue. The first paragraph or two must be capable of being used separately as a synopsis of the entire paper. Items of the bibliography should not be cited there unless absolutely necessary, in which case they must be identified by author and Journal, rather than by item number. Manuscripts, in triplicate, may be sent to any one of the editors. Please classify according to the scheme of Math. Reviews, Index to Vol. 39. All other communications should be addressed to the managing editor, or Elaine Barth, University of California, Los Angeles, California, 90024.

The Pacific Journal of Mathematics expects the author's institution to pay page charges, and reserves the right to delay publication for nonpayment of charges in case of financial emergency.

100 reprints are provided free for each article, only if page charges have been substantially paid. Additional copies may be obtained at cost in multiples of 50 .

The Pacific Journal of Mathematics is issued monthly as of January 1966. Regular subscription rate: $\$ 72.00$ a year $(6$ Vols., 12 issues). Special rate: $\$ 36.00$ a year to individual members of supporting institutions.

Subscriptions, orders for back numbers, and changes of address should be sent to Pacific Journal of Mathematics, 103 Highland Boulevard, Berkeley, California, 94708.

PUBLISHED BY PACIFIC JOURNAL OF MATHEMATICS, A NON-PROFIT CORPORATION

Printed at Kokusai Bunken Insatsusha (International Academic Printing Co., Ltd.), 8-8, 3-chome, Takadanobaba, Shinjuku-ku, Tokyo 160, Japan. 


\section{Pacific Journal of Mathematics}

\section{Vol. 61, No. 2 December, 1975}

Graham Donald Allen, Francis Joseph Narcowich and James Patrick Williams, An operator version of a theorem of Kolmogorov .......................

Joel Hilary Anderson and Ciprian Foias, Properties which normal operators share with normal derivations and related operators . . . . . . . . . . . . . . . . . . . . .

Constantin Gelu Apostol and Norberto Salinas, Nilpotent approximations and

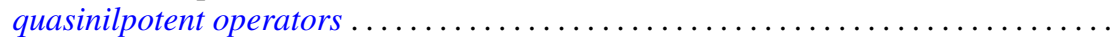

James M. Briggs, Jr., Finitely generated ideals in regular $F$-algebras . . . . . . . . . . .

Frank Benjamin Cannonito and Ronald Wallace Gatterdam, The word problem and power problem in 1-relator groups are primitive recursive ..................

Clifton Earle Corzatt, Permutation polynomials over the rational numbers ...........

L. S. Dube, An inversion of the $S_{2}$ transform for generalized functions . . . . . . . . . . William Richard Emerson, Averaging strongly subadditive set functions in unimodular

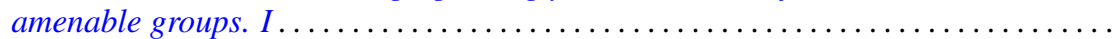

Barry J. Gardner, Semi-simple radical classes of algebras and attainability of

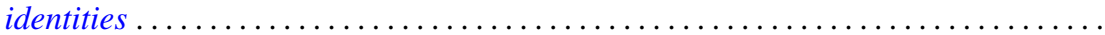

Irving Leonard Glicksberg, Removable discontinuities of A-holomorphic functions ....

Fred Halpern, Transfer theorems for topological structures . . . . . . . . . . . . . . .

H. B. Hamilton, T. E. Nordahl and Takayuki Tamura, Commutative cancellative

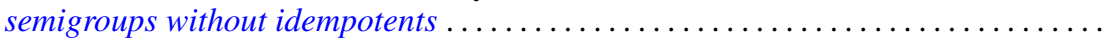

Melvin Hochster, An obstruction to lifting cyclic modules .....................

Alistair H. Lachlan, Theories with a finite number of models in an uncountable power

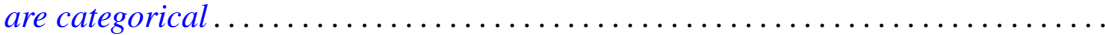

Kjeld Laursen, Continuity of linear maps from $C^{*}$-algebras . . . . . . . . . . . . .

Tsai Sheng Liu, Oscillation of even order differential equations with deviating arguments ....

Jorge Martinez, Doubling chains, singular elements and hyper- $Z$

Mehdi Radjabalipour and Heydar Radjavi, On the geometry of num Thomas I. Seidman, The solution of singular equations, I. Linear equations in Hilbert

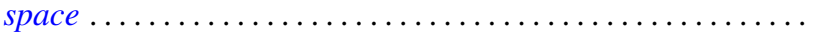

R. James Tomkins, Properties of martingale-like sequences ......

Alfons Van Daele, A Radon Nikodým theorem for weights on von Neumann

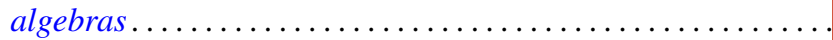

Kenneth S. Williams, On Euler's criterion for quintic nonresidues

Scott Andrew Wolpert, Noncompleteness of the Weil-Petersson metric for Teichmüller

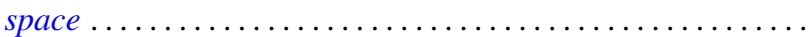

Volker Wrobel, Some generalizations of Schauder's theorem in locally convex

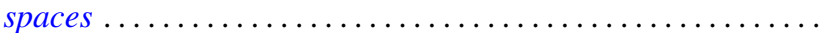

Kelly Denis McKennon, Corrections to: "Multipliers of type $(p, p)$ "; "Multipliers of type $(p, p)$ and multipliers of the group $L_{p}$-algebras"; "Multipliers and the

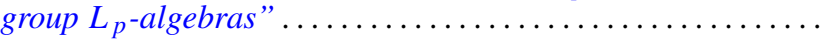

Andrew M. W. Glass, W. Charles (Wilbur) Holland Jr. and Stephen H. McCleary, Correction to: " $a *$-closures to completely distributive lattice-ordered

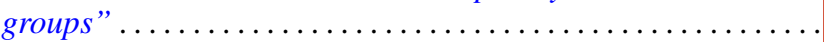

Zvi Arad and George Isaac Glauberman, Correction to: "A characteristic subgroup of

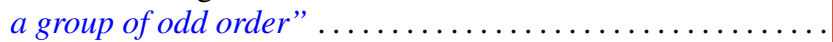

Roger W. Barnard and John Lawson Lewis, Correction to: "Subordination theorems

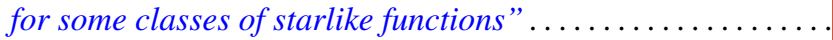

